\title{
Editorial: From imputation to impact
}

\section{Heather Joshi}

The topics included in this issue range from the imputation of missing data in longitudinal surveys to demonstrating that their results make a difference in the public arena - both challenges to our research field the world over. Along the way through these pages, the papers include studies of various intergenerational transmissions of social advantages and disadvantages, and social predictors of the mental health of adults. As it happens, three Australian longitudinal datasets feature in these contributions, suggesting that the creation and analysis of longitudinal data resources is thriving 'down under'.

Missing data is a particular challenge in longitudinal research due to survey attrition. The first paper by Panteha Hayati Rezvan, Katherine Lee and Julie Simpson deals with a technique for multiple imputation of missing data where it cannot be assumed that such information is missing at random. The technique explained here, the delta adjustment method, uses expert assessments in the construction of a sensitivity analysis around the bias that may be generated when data are missing not at random. Although this approach is applicable more generally, the authors offer an illustration using data from the Longitudinal Study of Australian Children. They estimate a range of possible bias in the association of maternal distress when a child is age 4-5 with that child's Total Difficulties score four years later, on the Strengths and Difficulties Questionnaire, allowing for the possibility that both variables may be subject to non-random survey loss.

The second article, by Jack Lam and Francisco Perales investigates the stress process theory of adult mental health. It is based on 15 years of data from the Household, Income and Labour Dynamics in Australia (HILDA). Mental health appears to be protected by living in a couple, particularly if married, and particularly in the case where one of them suffers from chronic illness. The relationship applies to both genders. This points to policy implications including the need to recognise the vulnerability of unpartnered people with chronic illness to mental health problems.

The relationship of adult mental health to adverse life events, explored in the paper by
Mandemakers and Kalmijn, uses data from the Netherlands Kinship Panel. The adverse events considered are partner loss (divorce/separation or death), death of a parent, unemployment and disability. The findings for partner loss echo patterns seen on the other side of the globe by Lam and Perales. This study adds to the picture by quantifying the cumulative and interactive impact of different sorts of troubles coming together.

The fourth paper, by Tiina Ristikari, Marko Merikukka and Mia Kristina Hakovirta, returns to the association of parents' adversity with offspring outcomes, in this case of young adults. This study is based on linked administrative records from the Finnish Cohort Study of people born in 1987. Adversity in childhood is represented by parents claiming social assistance for various timings and durations. Their offspring's outcomes to age 25 include educational failure, crime and teenage motherhood. Results confirm that early and persistent poverty has strong links with these problems in early adulthood.

By contrast, the paper by Francis Green, Samantha Parsons, Alice Sullivan and Richard Wiggins is concerned with the transmission of good fortune from one generation to the next. Taking data from the British Household Panel and the British 1970 Cohort, they investigate homogamy among people who have attended private schools. Women who have been to private schools are somewhat more likely than their state-educated counterparts to marry privately educated men. They are also more likely to have highly paid husbands. This contributes to the forces maintaining social immobility in Britain.

The Study Profile contributed by a team from Queensland University (Gita Mishra and nine colleagues) introduces the third Australian dataset this issue - the extension of the Australian Longitudinal Study of Women's Health to the second generation. MatCH (Mothers and their Children's Health) uses an internet or postal followup about the children's health, 'matched' to administrative sources about their education. As the data collection on the children was not completed until mid 2017, analysis has hardly begun, and its potential remains open. 
We close with a piece based on David Bell's keynote talk at the 2017 SLLS Conference in Stirling. Based on his experience of setting up HAGIS (Healthy Ageing in Scotland), he discusses the requirement that long-term studies demonstrate their utility to the public and policy maker in the form of something called 'impact'. As was recently reaffirmed in the ESRC's review of UK longitudinal resources, there is an expectation by funders that investments in such resource justify their existence by demonstrating 'impact', beyond the academic sphere. Bell expresses some scepticism about establishing the long-term scientific benefits from short-term evidence. SLLS tries to help promote policy engagement of longitudinal research through the activities of its Policy Group and media outreach. Another step towards the elusive goal may be to give explicit credit to the data resources when we write up the results of research based upon them. They cannot be taken for granted. 